\title{
Patterns of Presentation in Gonococcal Urethritis and Assessment of Antibiotic Sensitivity in Gonorrhea \\ Khawer Saleem ${ }^{1 *}$ and Waqar Azim ${ }^{2}$
}

${ }^{1}$ Department of Dermatology PNS Shifa Karachi, Pakistan

${ }^{2}$ Department of Pathology PNS Shifa Karachi, Pakistan

\begin{abstract}
Objective: To see various features of Gononcoccal Urethritis and assess the results of Gram stain and culture tests in cases of Gonorrhea and to see the antibiotic resistance pattern in them, in our local population (PNS Shifa hospital Karachi, Pakistan).
\end{abstract}

Design: Prospective case series.

Place and duration of study: Skin department of PNS Shifa Hospital Karachi, Pakistan. June 2004 to December 2007.

Materials and methods: The study was carried out in the outpatient dermatology department of PNS Shifa hospital Karachi during the period from June 2004 to Dec 2007. It was a prospective study. One hundred clinically suspected patients of Gonococcal urethritis were included in the study. Detailed history including history of sexual contact was taken. General and systemic physical examination was carried out in all the patients. Dermatological examination including examination of external genitalia was also done. In all these patients following investigations were carried out. Urine complete examination, complete blood counts, pus sample was collected from external urethral meatus by two sterile inoculating loops on to a clean glass slide for Gram staining which was seen under the microscope for the presence of gram-negative diplococci. The other loop was inoculated onto chocolate agar plate and was incubated in an atmosphere of $5 \% \mathrm{CO}_{2}$ at $37^{\circ} \mathrm{C}$ for $24-48 \mathrm{~h}$. The growths obtained were identified with colony morphology, Gram staining, oxidase reaction and rapid sugar fermentation. The Gonococci isolated were tested for antibiotic sensitivity using modified Kerby buer technique. The antibiotics included Penicillin G, Tetracycline, Cefotaxime, Cefuroxime, Ceftriaxone and Ciprofloxacin. HIV test was done in all patients. The results of two types of tests and their merits and demerits were assessed and compared..

Results: A total of 100 patients having urethral discharge and dysuria were studied. All the patients were males. The ages varied from 20 to 40 years, the average being 29.2 years +5.8 years. History of illicit sexual exposure was elicited in 43 $(43 \%)$ patients. The duration of period of symptoms ranged between 4 and 30 days with a mean of 12.8 days and a median of 12 days. The patients with gonorrhea presented with purulent discharge in $66(66 \%)$ cases, and dysuria in $49(49 \%)$ cases. No case had anal or oral symptoms. Examination showed a reddened external urethral meatus in 53(53\%) cases. Eighty seven (87\%) cases showed intracellular diplococci on direct microscopy of Gram stained smear. Sixty-two (62\%) cases showed growth of gonococci on culture of urethral pus. No case was seen to be HIV positive. Tetracycline resistance was seen in 34(55\%) strains. Penicillin resistance was seen in 25 (41\%) strains of Neisseria Gonorrhea. Five strains (11.5\%) showed resistance to Ciprofloxacin. No resistance was seen with Cephalosporins.

Conclusion: Gram staining and culture for Neisseria Gonorrhea are both useful diagnostic tests for Gonococcal urethritis. Drug resistance of Neisseria Gonorrhea is high with various antibiotics except for Cephalosporins stressing the need for culture based therapy and enthusiastic prevention of Gonorrhea in our society.

Keywords: Gonorrhea; Gram stain; Culture; Urethral discharge

\section{Introduction}

Gonorrhea is a common sexually transmitted disease, which is caused by Neisseria gonorrhea. This is transmitted between individuals by direct, usually sexual contact. It is the second most commonly reported communicable disease, with more than 350,000 cases reported annually [1]. In males the most common manifestation of infection is acute urethritis characterized by abrupt onset of dysuria and a purulent urethral discharge. The symptoms usually appear within 2 to 10 days of infection, but the incubation period is sometimes much longer, and some men never develop symptoms. The incidence of gonorrhea declined significantly in most developed countries during the 1980s, but remains common in some urban centers in the world. It is prevalent in many developing countries. The male: female ratio is 2:1 [2].

Neisseria gonorrhea is highly susceptible to adverse environmental influences such as temperature extremes and drying and it does not survive long outside its natural host, which is man. Infection with Gonorrhea increases the risk of becoming infected with HIV. This is likely due to weakening of the mucosal surface secondary to the Gonorrhea infection [3]. The Gram stain and the culture test are the two standard tests for Gonorrhoea [4]. The Gram stain involves placing a smear of the discharge on a slide where it is stained with Gram stain and examined for Gonococcal diplococci under a microscope. It has high sensitivity and specificity (over $90 \%$ in males) for the diagnosis [5]. The culture test is more reliable but takes longer. It involves taking a swab of the discharge, rolling it on to a culture plate, and incubating it under special laboratory

*Corresponding author: Dr. Khawer Saleem, Consultant Dermatologist, Ahalia Medical Center, Al Ain, UAE, Tel: 00971-50-4680689; E-mail: dr_khawer_saleem@yahoo.com

Received August 03, 2016; Accepted August 18, 2016; Published August 25 2016

Citation: Saleem K, Azim W (2016) Patterns of Presentation in Gonococcal Urethritis and Assessment of Antibiotic Sensitivity in Gonorrhea. J AIDS Clin Res 7: 613. doi: 10.4172/2155-6113.1000613

Copyright: ( 2016 Saleem K, et al. This is an open-access article distributed unde the terms of the Creative Commons Attribution License, which permits unrestricted use, distribution, and reproduction in any medium, provided the original author and source are credited. 
conditions for 16 to 48 hours to let the Gonococci multiply [6]. For the diagnosis of gonorrhea, appropriate sites for specimen collection depend to some extent on the age, sex and sexual practices of the individual and the clinical features of the infection. The commonest sample is pus from genitourinary passage. If standard culture media are available at the collection site, the specimen is directly inoculated and is placed in an atmosphere containing $5 \% \mathrm{CO}_{2}$ at $37^{\circ} \mathrm{C}$. It is then transported to laboratory. Gonococci are fastidious in their growth requirements. Most of the times these are present along with normal flora [7]. The culture media used are, therefore, both enriched and selective. For routine culture chocolate agar base medium of good quality with $9 \%$ Saponin lysed horse/sheep blood and inhibitors (Vancomycin to suppress the growth of Gram positive, Colistin to suppress Gram negative organisms, Nystatin to suppress yeast multiplication and Trimethoprim is added to inhibit swarming by Proteus spp.) [8]. Antibiotic treatment is an essential control measure for Gonorrhea. Antibiotic treatment is usually administered as a standard treatment of a single dose of antibiotic. The standard treatment regimen should cure more than $95 \%$ of cases. Antibiotic resistance in Neisseria gonorrhea, to agents used for treatment, has been a continuing problem. So a surveillance of antibiotic resistance with a change in treatment regimens when resistance occurs in $>5 \%$ of isolates is recommended. Antimicrobial resistance in Gonorrhea including both chromosomal resistance and plasmid mediated resistance has increased worldwide recently [9].

The aim of the study was to see the pattern of presentation, to assess the results of Gram stain and culture tests in cases of Gonorrhoea in our population (reporting to PNS Shifa hospital Karachi, Pakistan) and to see the antibiotic resistance pattern of Tetracyclines, Penicillins, Quinolones and Cephalosporins in them.

\section{Materials and Methods}

The study was carried out in the outpatient dermatology department of PNS Shifa hospital Karachi, Pakistan, during the period from June 2004 to Dec 2007. It was a prospective case study. One hundred clinically suspected patients of Gonococcal urethritis were included in the study. Only male patients of all ages were included in the study. Patients who had received systemic treatment for their complaints and those having other inter-current illnesses were excluded from the study. Detailed history of symptoms and history of sexual contact was taken. General and systemic physical examination was carried out in all the patients. Local examination including examination of external genitalia, testis, oral cavity, eyes and anus was also done. In all these patients investigations carried out were urine complete examination and complete blood counts along with ESR were done. Genitalia were cleaned up with normal saline and a slight pressure on penis was exerted to exude a drop of pus at external urethral meatus. The pus was collected by two sterile inoculating loops. One loop was rolled up on a clean glass slide to prepare a thin and homogenous film which was allowed to air dry before it was Gram stained. This was seen under microscope for the presence of Gram-negative diplococci. The other loop was inoculated onto a $1 \mathrm{~cm}$ circular area of the chocolate agar plate. The chocolate agar contained Vancomycin and was incubated in an atmosphere of 5\% $\mathrm{CO}_{2}$ at $37^{\circ} \mathrm{C}$ for $24-48 \mathrm{~h}$. The growths obtained were identified with colony morphology, Gram staining, oxidase reaction and rapid sugar fermentation. HIV test was performed in all the patients using Elisa method. The Gonococci isolated were tested for antibiotic sensitivity using modified Kerby buer technique [10]. The antibiotics included Penicillin G, Tetracycline, Cefotaxime, Cefuroxime, Ceftriaxone and Ciprofloxacin. Culture testing was done by growing bacteria on a nutrient plate and then exposing them to known amounts of an antibiotic to determine the bacterial susceptibility to the antibiotic. The results of two types of tests and their merits and demerits were assessed.

\section{Results}

A total of 100 patients having urethral discharge were studied. All the patients were males. The age varied from 20 to 40 years, the average being 29.2 years +5.8 years. Majority of patients seen were in the age group of $21-30$ years (60\%). History of illicit sexual exposure could be elicited in $36(36 \%)$ patients whereas rest of the patients denied it. There was history of solitary sexual exposure in $26(72.3 \%)$ patients and multiple exposures in $10(27.7 \%)$ patients. The duration of symptoms ranged between 4 and 30 days with a mean of 12.8 days and a median of 12 days. The patients with Gonococcal Urethritis presented with purulent discharge (both frank and scanty) in 66 (66\%) cases, and dysuria in $49(49 \%)$ cases, $2(2 \%)$ cases had unilateral swelling of testis, 1 (1\%) patient had fever and lower abdominal pain, $23(23 \%)$ patients had erythema and swelling at external urethral meatus. No case had anal, eye or oral symptoms. Thirty four (34\%) cases had an elevated white blood cell (WBC) count, in the range of 10,000-15,000/ $\mu \mathrm{L}$. Forty one $(41 \%)$ cases had elevated erythrocyte sedimentation rate (ESR) in the range of $20-50 \mathrm{~mm}$ fall at the end of It hour. Eighty seven $(87 \%)$ cases showed intracellular diplococci on direct microscopy of Gram stained smear. Sixty-two (62\%) cases showed growth of gonococci on culture of urethral pus. No case was seen to be HIV positive. Tetracycline resistance was seen in 34(55\%) strains. Penicillin resistance was seen in 25 (41\%) strains of Neisseria Gonorrhea. Five strains (11.5\%) showed resistance to Ciprofloxacin. No resistance was seen with Cephalosporins.

\section{Discussion}

The duration of symptoms in our study ranged between 4 and 30 days with a mean of 12.8 days and a median of 12 days. Some others studies in this regard have shown duration of symptoms to be 8 days to 2 weeks [11]. Men with Gonococcal urethritis may present with any combination of symptoms [12]. In our study the patients with gonorrhea presented with purulent discharge (both frank and scanty) in $66(66 \%)$ cases, and dysuria in $49(49 \%)$ cases, 2 (2\%) cases had unilateral swelling of testis, $1(1 \%)$ patient had fever and lower abdominal pain, 23 (23\%) patients had erythema and swelling at external urethral meatus (Table 1). The discharge was mostly present spontaneously at

\begin{tabular}{|l|c|}
\hline Age & $\begin{array}{c}\mathbf{2 0 - 4 0} \text { years.Average-29.2 } \\
\text { years+5.8 years. } \\
\mathbf{6 0 \%} \text { - Maximum: 21-30 years }\end{array}$ \\
\hline Gender & \begin{tabular}{c} 
Males \\
\hline H/O Illicit Sexual Contact
\end{tabular} \\
\hline Duration of Symptoms & $\begin{array}{c}4-30 \text { days } \\
\text { Mean-12.8 days } \\
\text { Median-12 days }\end{array}$ \\
\hline Purulent Discharge & $66 \%$ \\
\hline Dysuria & $49 \%$ \\
\hline Epididymitis & $2 \%$ \\
\hline Fever \& Lower abdominal pain & $1 \%$ \\
\hline Erythema at External urethral meatus & $23 \%$ \\
\hline $\begin{array}{l}\text { Elevated WBC count, in the range of 10,000- } \\
15,000 / \mu \mathrm{L} .\end{array}$ & $34 \%$ \\
\hline $\begin{array}{l}\text { Elevated ESR in the range of } 20-50 \text { mm fall at } \\
\text { the end of Ist } h\end{array}$ & $41 \%$ \\
\hline Gonococci seen on Gram stain of Smear & $87 \%$ \\
\hline Positive Gonococcal Culture & $62 \%$ \\
\hline
\end{tabular}

Table 1: Presentation pattern in gonorrhea patients, $n=100$. 
the urethral meatus and in a few cases was elicited by exerting a slight pressure on penis. The discharge was scanty and copious, purulent or muco-purulent in consistency. In another such study discharge was present in 82 percent of cases and dysuria in 53 percent [13]. Unilateral testicular pain and swelling may be the sole presenting complaints of men with epididymitis, with concomitant urethritis often discovered during the history and physical examination as it was seen in $2(2 \%)$ cases in our study. No extra genital symptoms were seen in our study. History of sexual exposure was scarce as it could be elicited only in $36 \%$ cases which may be due to considering of illicit sexual contact as a taboo in this part of world. In our study the highest incidence $(60 \%)$ was found to be in the age group of 21-30 years which is almost similar to certain other studies in this regard [14]. All the cases in our study had urethritis. No case had oral or anal symptoms.

Specific culture of a swab from the site of infection is a criterion standard for diagnosis at all potential sites of Gonococcal infection. In male patients with urethritis, the diagnosis can be made by direct microscopy of stained smears of urethral discharge [15]. Cultures are particularly useful when the clinical diagnosis is unclear as in asymptomatic males and in females, when a failure of treatment has occurred, when contact tracing is problematic, and when legal questions arise. Following culture of specimens obtained from the genital tract using a highly selective medium, a presumptive diagnosis can be made based on colony morphology; Gram stain and the detection of cytochrome $\mathrm{C}$ oxidase [16]. A number of swab types are suitable for collecting specimens of $N$. gonococci. These include serum/albumincoated swabs, calcium-alginate swabs and some modern rayon fibertipped swabs. Sterile inoculating loops can also be used. It is always preferable to collect two swabs, one for microscopic examination and the other for culture. If standard culture media are available at the collection site, the specimen can be inoculated at the site and placed in an atmosphere containing $5 \% \mathrm{CO}_{2}$ at $37^{\circ} \mathrm{C}$. The inoculated plates can then be transported at a convenient time [17]. In our study the positive Gram stained smears were seen in $87 \%$ cases of suspected Gonococcal urethritis. It was easy to perform the test in OPD or on the bedside of the admitted patient. Results were received quickly. The cost of this test was quite low (Table 2). This test was found to be quite accurate for men but is considered not good in women. It is generally known that only one in two women with gonorrhea have a positive stain [18]. In one study, a Gram stain diagnosed 94 percent of cases in symptomatic men however the sensitivity dropped to 81 percent in asymptomatic subjects. Some other reports have shown that performance of Gram stain in a man is similar to that of culture, with sensitivities of 89 to 94 percent and specificities of 94 to $97 \%$ [19]. Thus, in symptomatic men, urethral Gram stain is sensitive, specific, and cost effective.

\begin{tabular}{|l|l|l|}
\hline \multicolumn{1}{|c|}{ Microscopy } & \multicolumn{1}{c|}{ Culture } \\
\hline $\begin{array}{l}\text { Time } \\
\text { Required }\end{array}$ & Rapid & 24-48 $\mathrm{h}$ \\
\hline Cost & Inexpensive & Moderately Expensive \\
\hline Utility & $\begin{array}{l}\text { Ineffective for females, } \\
\text { rectal and pharyngeal } \\
\text { samples }\end{array}$ & Effective for all types of samples \\
\hline Level of use & In OPD and on bed side & Laboratory facilities are required \\
\hline $\begin{array}{l}\text { Ease of } \\
\text { performance }\end{array}$ & Easy & Moderate \\
\hline Equipment & Light microscope & $\begin{array}{l}\text { Transport Medium, Culture medium, } \\
\text { incubator, light microscope, candle jar }\end{array}$ \\
\hline $\begin{array}{l}\text { Antibiotic } \\
\text { sensitivity }\end{array}$ & Can not be determined & Can be determined \\
\hline
\end{tabular}

Table 2: Comparison of gram stain and culture for detection of Niesseria gonorrhoea.
However, if the Gram strain is negative in an asymptomatic man, additional testing should be performed if Gonorrhea is suspected due to risk factors. Recently developed tests using cycling probe technology, such as examination of urine by PCR, allow diagnosis to be made without the need for culture. However, these tests are costly and culture is essential for surveillance of antimicrobial susceptibility. The positivity of the culture in our study was found to be $62 \%$. Culture test was more difficult to perform than the Gram stain smear and required the patient to be referred to the laboratory as the availability of transport media was sparse and difficult. The culture test was more reliable but required 24-48 hrs for the results. The cost of the test was higher than the smear test. Costs for culture are moderate compared with more expensive DNA amplification techniques (Table 3).

Over the last decade, strains of Neisseria gonorrhea have been reported to develop high levels of resistance against several antimicrobial agents which have been used previously for the treatment of gonorrhea [20]. Gonorrhea has progressively developed resistance to various antibiotics in our population also. Fortunately the sensitivity to Cephalosporins is still preserved. No patient had positive HIV test in our study. However high level of HIV positivity was found in cases of Gonorrhea in certain other studies. Khopakar et al reported $14 \%$ positivity rate in all STD cases and Bhushanam et al 2.3\% [21]. The resistance to Cephalosporins is also being reported from certain other areas of world [22]. Furthermore the frequent travel is also spreading the multi-drug resistant strains across the world. The reason for resistance seems to be the ability of gonococcus to mutate and develop efflux pump, a structure that evolved in several types of bacteria to pump antimicrobial compounds out of the organism before they can do any harm [23]. The emergence of cephalosporin resistance would substantially limit treatment options and would be a significant public health concern, in particular because untreated gonorrhea can prompt to costly and severe public health consequences. The untreated Gonorrhea can lead to urethral strictures, pelvic inflammatory disease and infertility both in males and females. In USA there are approximately close to 700,000 cases of Gonorrhea each year. The drug resistant strains of gonorrhea were 1 in 10 cases of sexually transmitted diseases in 2010 in Europe [24]. In this perspective it becomes all the more imperative to develop newer effective antibiotics against the organism and stress upon the prevention of disease in our society. A major challenge to monitoring emerging antimicrobial resistance of $N$. gonorrhea is the substantial decline in the use of gonorrhea culture by many clinicians, as well as the reduced capability of many laboratories to perform gonorrhea culture techniques required for antibiotic susceptibility testing. Culture testing is when the bacteria is first grown on a nutrient plate and is then exposed to known amounts of an antibiotic to determine the bacteria's susceptibility to the antibiotic. The decline in culture testing results from an increased use of newer nonculture-based laboratory technology, such as a diagnostic test called the Nucleic Acid Amplification Test (NAAT). Currently, there is no well-studied reliable technology that allows for antibiotic susceptibility testing from non-culture specimens. Increased laboratory culture capacity is needed. CDC recommends that all state and local health department labs maintain or develop the capacity to perform gonorrhea

\begin{tabular}{|c|c|}
\hline Drug & Sensitivity \\
\hline Tetracyclines & $34(55 \%)$ \\
\hline Penicillins & $25(41 \%)$ \\
\hline Quinolones & $5(11.5 \%)$ \\
\hline Cephalosporins & Nil \\
\hline
\end{tabular}

Table 3: Sensitivity pattern of Neisseria gonorrhoea. 
Citation: Saleem K, Azim W (2016) Patterns of Presentation in Gonococcal Urethritis and Assessment of Antibiotic Sensitivity in Gonorrhea. J AIDS Clin Res 7: 613. doi: 10.4172/2155-6113.1000613

Page 4 of 4

culture [25]. CDC's 2015 STD Treatment Guidelines now recommend only one regimen of dual therapy for the treatment of gonorrheathe injectable cephalosporin ceftriaxone, plus oral azithromycin. Dual therapy is recommended to address the potential emergence of gonococcal cephalosporin resistance [26]. A safe and effective vaccine for gonorrhea would greatly decrease the urgency of developing newer antibiotics for N.Gonorrhoea. Dr. Cynthia Cornelissen, an NIAIDfunded investigator at Virginia Commonwealth University, is working toward that goal [27].

\section{Conclusion}

Gram staining and culture for Neisseria gonorrhea are both useful diagnostic tests for Gonococcal urethritis. Drug resistance of Neisseria Gonorrhea is high with various antibiotics except for Cephalosporins stressing the need for culture based therapy and development of newer antibiotics along with enthusiastic prevention of Gonorrhea in our society.

\section{References}

1. Murray PR, Ken SR, Michael AP (2009) Medical Microbiology (6th ed) Mosby/ Elsevier, Philadelphia.

2. Marrazzo JM, Apicell MA (2015) Mandell, Douglas and Bennett's principles and practice of infectious diseases, 8th edition. Elsevier Saunders 2: 214.

3. Horner P (2007) Asymptomatic men: Should they be tested for urethritis? Sex Transm Infect 83: 81-84.

4. Laboratory diagnosis of gonorrhea (1999) WHO Regional Publication SouthEast Asia 33.

5. Chan EL, Kingston MA, Carlin EM (2004) The laboratory diagnosis of gonorrhoea and syphilis infection. J Fam Plann Reprod Health Care 30: 126127

6. Bachmann LH, Manhart LE, Martin DH, Seña AC, Dimitrakoff J, et al. (2015) Advances in the understanding and treatment of male urethritis. Clin Infect Dis 61 Suppl 8: S763-769.

7. National guideline on the diagnosis and treatment of gonorrhea in adults 2005 (2005) British Association for Sexual Health and HIV (BASHH), London 9: 36.

8. Groopman, Jerome (2012) Sex and the superbug. The New Yorker 138: 26-31.

9. Deguchi T, Nakane K, Yasuda M, Maeda S (2010) Emergence and spread of drug resistant Neisseria gonorrhoeae. J Urol 184: 851-858.

10. Bonev B, Hooper J, Parisot J (2008) Principles of assessing bacterial susceptibility to antibiotics using the agar diffusion method. J Antimicrob Chemother 61: 1295-1301.

11. Harrison WO, Hooper RR, Wiesner PJ, Campbell AF, Karney WW, et al. (1979)
A trial of minocycline given after exposure to prevent gonorrhea. $\mathrm{N}$ Engl $\mathrm{J}$ Med 300: 1074-1078.

12. Smith L, Angarone MP (2015) Sexually Transmitted Infections. Urol Clin North Am 42: 507-518.

13. Sherrard J, Barlow D (1996) Gonorrhoea in men: Clinical and diagnostic aspects. Genitourin Med 72: 422-426.

14. Gupta CM, Sanghi S, Sayal SK, Das AL, Prasad GK (2001) Clinical and bacteriological study of urethral discharge. Indian J Dermatol Venereol Leprol 67: 185-187.

15. Desai M, Woodhall SC, Nardone A, Burns F, Mercey D, et al. (2015) Active recall to increase HIV and STI testing: A systematic review. Sex Transm Infect 91: 314-323.

16. Janda WM, Knapp JS. Manual of clinical microbiology (8th ed) American Society for Microbiology, Washington, DC

17. Chorba T, Tao G, Irwin K (2004) Urologic diseases in America. Sexually Transmitted Diseases 233-279.

18. Workowski KA, Levine WC (2002) Selected topics from the Centers for Disease Control and Prevention Sexually Transmitted Diseases Treatment Guidelines 2002. HIV Clin Trials 3: 421-433.

19. Frenkl T, Potts J (2006) Sexually transmitted infections: Part II - Associated Vaginitides and Urethritides. AUA Update 25: 17-19.

20. Catharine Paddock (2016) Antibiotic resistance raising the specter of 'untreatable gonorrhea.

21. Melanie MT, Julia AS, Bruce WF (2013) Gonorrhea Infections Diagnosed Among Persons Living With HIVIAIDS. J Acquir Immune Defic Syndr 64: 115120.

22. Robert DK, Harvey A, Papp JR (2014) Neisseria gonorrhoeae Antimicrobial Susceptibility Surveillance - The Gonococcal Isolate Surveillance Project, 27 Sites, United States. Morbidity and Mortality Weekly Report, CDC.

23. Health Protection Agency (2006) The gonococcal resistance to antimicrobials surveillance programme: Annual report 2005.

24. Cornelissen $\mathrm{CN}$ (2008) Identification and characterization of gonococcal iron transport systems as potential vaccine antigens. Future Microbiol 3: 287-298.

25. Long F, Rouquette-Loughlin C, Shafer WM, Yu EW (2008) Functional cloning and characterization of the multidrug efflux pumps NorM from Neisseria gonorrhoeae and YdhE from Escherichia coli. Antimicrob Agents Chemother. 52: 3052-3060.

26. Chan $\mathrm{CH}$, McCabe CJ, Fisman DN (2011) Core groups, antimicrobial resistance and rebound in gonorrhoea in North America. Sex Transm Infect.

27. Centers for Disease Control and Prevention (2015) Sexually transmitted disease surveillance 2014. Department of Health and Human Services, Atlanta, USA. 